\title{
Application des harmoniques générées dans un jet de gaz: mesure de sections efficaces d'ionisation des états excités de I'hélium
}

\author{
D. Descamps, A. L'Huillier, C. Lyngå, C.-G. Wahlström, \\ M. Gisselbrecht* et M. Meyer*
}

\author{
Department of Physics, Lund Institute of Technology, P.O. Box 118, 22100 Lund, Sweden \\ * LURE, Laboratoire pour l'Utilisation du Rayonnement Électromagnétique, bâtiment 209D, \\ 91405 Orsay, France
}

\begin{abstract}
Résumé : Les sections efficaces d'ionisation des états excités de 1'hélium $1 \mathrm{~s} 2 \mathrm{p}{ }^{1} \mathrm{P}$ et $1 \mathrm{~s} 3 \mathrm{p}{ }^{1} \mathrm{P}$ ont été mesurées au voisinage du seuil d'ionisation en observant la saturation de l'ionisation. Les états excités de l'hélium sont préparés par l'absorption résonnante d'une harmonique d'ordre élevée produite par un laser picoseconde accordable. Ces états sont ensuite ionisés par un faisceau sonde en absorbant un photon. La modification de la fréquence du faisceau sonde, du proche infrarouge à l'ultraviolet, nous a permis de déterminer la dépendance de la section efficace d'ionisation en fonction de l'énergie de l'électron arraché. Les résultats expérimentaux confirment quantitativement les travaux théoriques effectués auparavant.
\end{abstract}

\section{INTRODUCTION}

La génération d'harmonique dans un jet de gaz permet de disposer d'une source XUV sur une large bande spectrale, couvrant même aujourd'hui le domaine des X mous [1]. Les caractéristiques spatiales et temporelles de cette source fortement corrélées à celles du laser en font une source très attractive pour nombres d'applications tel que la spectroscopie atomique, la physique des surfaces, la biologie ...

Après une première expérience de type pompe-sonde relative à la mesure de la durée de vie des états excités $1 \mathrm{~s} 2 \mathrm{p}{ }^{\mathrm{l}} \mathrm{P}(\mathrm{E}=21.22 \mathrm{eV}$ soit $\lambda=58.32 \mathrm{~nm})$ et $1 \mathrm{~s} 3 \mathrm{p}{ }^{1} \mathrm{P}(\mathrm{E}=23.09 \mathrm{eV}$ soit $\lambda=53.60 \mathrm{~nm}) \mathrm{de}$ l'hélium [2], une seconde expérience a été effectuée en vue de mesurer les sections efficaces d'ionisation de ces états excités au voisinage du seuil d'ionisation. Cette expérience a permis de valider les calculs récents de section efficace d'ionisation effectués par T. Chang et al. [3] et $Z$. Felfli et $S$. Manson [4] pour les états excités $2 p$ et $3 p$ de l'atome d'hélium et de montrer les potentiels de la source XUV développée au département de physique atomique de l'université de Lund.

\section{IONISATION À DEUX PHOTONS DE L'ATOME D'HÉLIUM}

L'excitation des états $2 p$ et $3 p$ de l'hélium est réalisée par les harmoniques d'ordre 13 et 14 (harmonique 7 du fondamental doublé en fréquence) générées dans un jet de krypton par un laser hybride coloranttitane:saphir. Outre une large accordabilité $(50 \mathrm{~mm})$, ce laser délivre des impulsions de $50 \mathrm{~mJ}$ sous une durée de 70 picosecondes permettant d'obtenir des conditions d'intensité optimum $\left(\mathrm{I} \sim 10^{14} \mathrm{~W} / \mathrm{cm}^{2}\right)$ pour la génération d'harmonique. Une des caractéristiques importantes de ce laser picoseconde est la faible largeur spectrale des impulsions produites $(\Delta \lambda<1 \AA)$ permettant de générer une source XUV $(\Delta \lambda=0.05 \AA)$ très efficace pour l'excitation de niveaux atomiques. La sélection d'une seule harmonique est effectuée par un spectromètre XUV constitué d'un réseau sphérique associé a une fente. Après avoir peuplé le niveaux excités de l'hélium ( $2 p$ ou $3 p$ ) par l'absorption d'un photon UV, nous réalisons l'ionisation avec un laser Nd:YAG dont on a doublé, triplé ou quadruplé la fréquence. Les ions $\mathrm{He}^{+}$produits sont ensuite détectés par un spectromètre à temps de vol.

\section{SATURATION DE L'IONISATION ET MESURE ABSOLUE DE LA SECTION EFFICACE D'IONISATION}

Indépendamment du nombre d'atomes excités produits lors de la première étape, il est possible de déterminer expérimentalement la section efficace d'ionisation des états excités peuplés. En effet, pour un pompage fixé, nous observons une saturation du nombre d'ions produits en augmentant l'énergie du 
faisceau sonde (cf. Fig. 1). Cette saturation dépend directement de la section efficace d'ionisation. A partir des équations de population, on montre que le nombre d'ions $\mathrm{He}^{+}$créés par l'ionisation à deux photons est décrit par l'expression suivante:

$$
\mathrm{N}_{\mathrm{He}^{+}}=\int \rho_{\mathrm{He}^{+}} \mathrm{dV}=\rho_{\mathrm{He}} \sigma_{\mathrm{abs}} \mathrm{e}^{-\Delta \tau / \tau f}\left[\int \mathrm{R}_{\text {pompe }}\left(1-\mathrm{e}^{-\sigma_{\text {ion }} \mathrm{R}_{\text {sonde }}}\right) \mathrm{dV}\right]
$$

où $\rho$ est la densité d'ions ou d'atomes d'hélium, $\sigma_{\mathrm{abs}}$ et $\sigma_{\text {ion }}$ sont respectivement les sections efficaces d'absorption et d'ionisation de l'hélium, $\Delta \tau$ est le retard temporel entre le faisceau pompe et le faisceau sonde, $\tau$ est la durée de vie de l'état excité peuplé et $R_{\text {pompe(sonde) }}$ sont les nombres de photons pompe(sonde) par unité de surface.

Moyennant la prise en compte des effets de volume qui modifie la saturation du signal enregistré, nous avons pu déterminer à partir de l'expression (1), la valeur absolue des sections efficaces d'ionisation des états $3 p$ et $2 p$ pour différentes longueurs d'onde du faisceau sonde. La figure 2 présente l'ensemble des sections efficaces d'ionisation mesurées pour l'atome d'hélium ainsi que les calculs théoriques. Les résultats obtenus sont en très bon accord avec les travaux théoriques et confirment la forte dépendance, au voisinage du seuil d'ionisation, de la section efficace d'ionisation avec l'énergie cinétique de l'électron arraché.

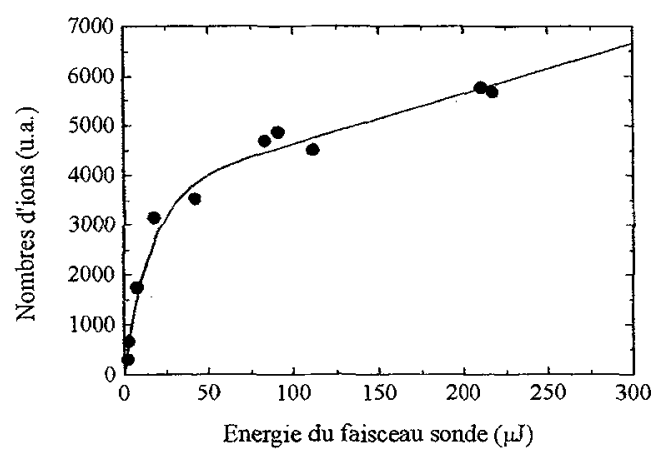

Figure 1 : Saturation de l'ionisation de l'état excité $1 \mathrm{~s} 2 \mathrm{p}{ }^{1} \mathrm{P}$ à la longueur d'onde sonde de $355 \mathrm{~nm}$.

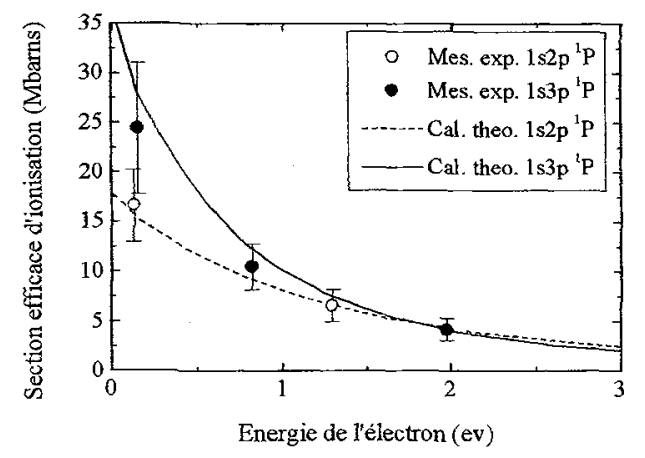

Figure 2 : Comparaison des sections efficaces d'ionisation mesurées et calculées.

\section{CONCLUSION}

Nous avons observé expérimentalement la dépendance de la section efficace d'ionisation d'états excités avec l'énergie de l'électron arraché. En modifiant la polarisation des faisceaux pompe et sonde, il est possible de déterminer les sections efficaces d'ionisation partielles vers les différents états du continum. De manière plus générale, ces résultats montrent clairement l'intérêt de la génération d'harmonique comme source XUV pour la spectroscopie atomique ou moléculaire. La méthode utlisée ici pour l'hélium peut être facilement transposée à l'étude d'un autre atome ou d'une molécule.

\section{Références}

[1] C. Spielmann et al., Science, Vol. 278, p. 661; October 1997.

Z. Chang et al., Opt. Lett., Vol. 79, No. 16, p. 2967, October 1997.

[2] J. Larson et al., J. Phys. B, Vol. 28, L53, 1995.

[3] T. N. Chang and T. N. Fang, Phys. Rev. A, Vol. 52, p. 2658, 1995.

[4] Z. Felfli and S. Manson, private communication, 1996. 